\title{
LTI Approximations of Slightly Nonlinear Systems: Some Intriguing Examples
}

\author{
Martin Enqvist, Lennart Ljung \\ Division of Automatic Control \\ Department of Electrical Engineering \\ Linköpings universitet, SE-581 83 Linköping, Sweden \\ WWW: http://Www. control.isy.liu.se \\ E-mail: maren@isy.liu.se, ljung@isy.liu.se
}

29th December 2005

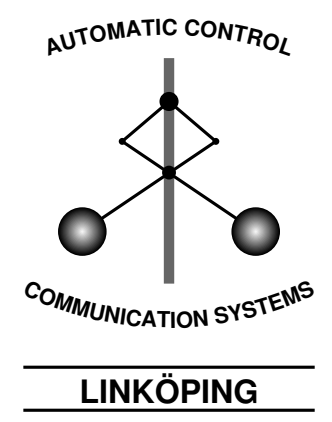

Report no.: LiTH-ISY-R-2655

Submitted to NOLCOS 2004 in Stuttgart, Germany.

Technical reports from the Control \& Communication group in Linköping are available at http://www.control.isy.liu.se/publications. 


\begin{abstract}
Approximations of slightly nonlinear systems with linear time-invariant (LTI) models are often used in applications. Here, LTI models that are optimal approximations in the mean-square error sense are studied. It is shown that these models can be very sensitive to small nonlinearities. Furthermore, the significance of the distribution of the input process is discussed. From the examples studied here, it seems that LTI approximations for inputs with distributions that are Gaussian or almost Gaussian are less sensitive to small nonlinearities.
\end{abstract}

Keywords: System identification, Nonlinear systems, Linearization 


\title{
LTI Approximations of Slightly Nonlinear Systems: Some Intriguing Examples
}

\author{
Martin Enqvist, Lennart Ljung
}

$2005-12-29$

\begin{abstract}
Approximations of slightly nonlinear systems with linear time-invariant (LTI) models are often used in applications. Here, LTI models that are optimal approximations in the mean-square error sense are studied. It is shown that these models can be very sensitive to small nonlinearities. Furthermore, the significance of the distribution of the input process is discussed. From the examples studied here, it seems that LTI approximations for inputs with distributions that are Gaussian or almost Gaussian are less sensitive to small nonlinearities.
\end{abstract}

\section{Introduction}

It is very common to approximate nonlinear systems using linear models. This approximation can be done in many ways. For example, differentiation can be used to linearize a nonlinear system description locally, or some kind of linear equivalent of a nonlinear system can be derived for a particular input. Here, we will investigate the latter of these two approaches.

More specifically, we will discuss some examples of linear model estimates obtained by system identification using input and output data from nonlinear systems. The system identification method that will be used here is the prediction-error method (Ljung, 1999), and we will only investigate its behavior when the number of measurements tends to infinity.

Consider a parameterized linear time-invariant (LTI) output error (OE) model

$$
y(t)=G(q, \theta) u(t)+e(t),
$$

where $\theta$ is a parameter vector and where $q$ denotes the shift operator, $q u(t)=$ $u(t+1)$. The signals $u(t), y(t)$ and $e(t)$ are the system input, output and noise, respectively. It is assumed that $G(q, \theta)$ is stable.

It can be shown (Ljung, 1978) that the prediction-error parameter estimate under rather general conditions will converge to the parameters that minimize a mean-square error criterion $\mathrm{E}\left((y(t)-G(q, \theta) u(t))^{2}\right)$. Here, $\mathrm{E}(x)$ denotes the expected value of the random value $x$. With this result in mind, it is interesting to investigate what can be said about the mean-square error optimal LTI approximation of a general, possibly nonlinear, system. More specifically, we are interested in the model that minimizes $\mathrm{E}\left((y(t)-G(q) u(t))^{2}\right)$ with respect to all stable and causal models $G(q)$. The mean-square error optimal model 
$G_{0, O E}(q)$ will here be called the Output Error Linear Time-Invariant Second Order Equivalent (OE-LTI-SOE).

LTI approximations of nonlinear systems can be studied in many frameworks. The idea of deriving an LTI approximation by differentiation of a nonlinear system is used, for example, by Mäkilä and Partington (2003). They study LTI approximations of nonlinear systems for $l_{\infty}$-signals and use the notion of Fréchet derivatives to derive some of the approximations. Related material can be found in Partington and Mäkilä (2002), Mäkilä (2003a) and in Mäkilä (2003b). LTI approximations for deterministic signals are also discussed in Sastry (1999) and in Horowitz (1993).

Furthermore, LTI approximations can also be studied in a stochastic framework. There has been a wide interest in understanding how a static nonlinearity can be linearized for a stochastic input signal. Many results in this area are directly or indirectly related to Bussgang's classic theorem about Gaussian signals (see Bussgang (1952) or, for example, Papoulis (1984)).

Bussgang (1952) has shown that the cross-covariance function between the output and the input of a static nonlinear function is a scaled version of the input covariance function if the input is Gaussian. Hence, the following theorem holds.

\section{Theorem 1.1 (Bussgang)}

Let $y(t)$ be the output from a static nonlinearity $f$ with a Gaussian input $u(t)$, i.e., $y(t)=f(u(t))$. Assume that the expectations $\mathrm{E}(y(t))=\mathrm{E}(u(t))=0$. Then

$$
R_{y u}(\tau)=b_{0} R_{u}(\tau),
$$

where $R_{y u}(\tau)=\mathrm{E}(y(t) u(t-\tau)), R_{u}(\tau)=\mathrm{E}(u(t) u(t-\tau))$ and $b_{0}=\mathrm{E}\left(f^{\prime}(u(t))\right)$.

Bussgang's theorem has turned out to be very useful for the theory of Hammerstein and Wiener system identification (Billings and Fakhouri, 1982; Korenberg, 1985; Bendat, 1998).

LTI approximations of nonlinear systems are discussed also by Pintelon and Schoukens (2001). They use the term related linear system for the mean-square error optimal LTI system and view the part of the output signal that this system cannot explain as a nonlinear distortion. Related material can be found in Pintelon et al. (2001), Pintelon and Schoukens (2002) and Schoukens et al. (2003). Schoukens et al. (2002) have also discussed benefits and drawbacks of different input signals for LTI approximations.

\section{Output Error LTI-SOEs}

In this paper, we will only consider nonlinear systems with input and output signals that have certain properties. These signal assumptions are listed here.

Assumption A1. Assume that

(i) The input $u(t)$ and output $y(t)$ are real-valued stationary stochastic processes with $\mathrm{E}(u(t))=\mathrm{E}(y(t))=0$.

(ii) There exist $K>0$ and $\alpha, 0<\alpha<1$ such that the second order moments $R_{u}(\tau)=\mathrm{E}(u(t) u(t-\tau)), R_{y u}(\tau)=\mathrm{E}(y(t) u(t-\tau))$ and $R_{y}(\tau)=\mathrm{E}(y(t) y(t-$ 


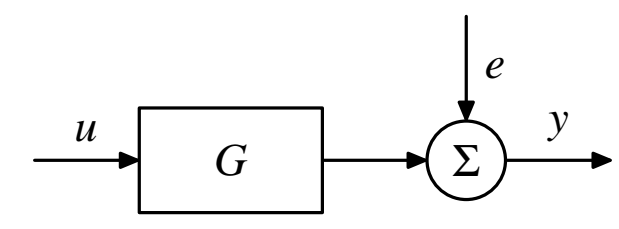

Figure 1: The output error model.

$\tau)$ ) satisfy

$$
\begin{aligned}
\left|R_{u}(\tau)\right|<K \alpha^{|\tau|}, & \forall \tau \in \mathbb{Z}, \\
\left|R_{y u}(\tau)\right|<K \alpha^{|\tau|}, & \forall \tau \in \mathbb{Z}, \\
\left|R_{y}(\tau)\right|<K \alpha^{|\tau|}, & \forall \tau \in \mathbb{Z} .
\end{aligned}
$$

(iii) The z-spectrum $\Phi_{u}(z)$ (i.e., the z-transform of $R_{u}(\tau)$ ) has a canonical spectral factorization

$$
\Phi_{u}(z)=L(z) r_{u} L\left(z^{-1}\right),
$$

where $L(z)$ and $1 / L(z)$ are causal transfer functions that are analytic in $\{z \in \mathbb{C}:|z| \geq 1\}, L(+\infty)=1$ and $r_{u}$ is a positive constant.

As mentioned above, we will here only consider output error models (Ljung, 1999). The structure of an output error model is shown in Figure 1.

Using only output error models, the mean-square error optimal LTI approximation of a certain nonlinear system is simply the causal and stable LTI model $G_{0, O E}$ that minimizes $\mathrm{E}\left((y(t)-G(q) u(t))^{2}\right)$. This model is often called the Wiener filter for prediction of $y(t)$ from $(u(t-k))_{k=0}^{\infty}$ (Wiener, 1949). However, in order to avoid any ambiguities we will instead call $G_{0, O E}$ the Output Error LTI Second Order Equivalent (OE-LTI-SOE) of the nonlinear system. Hence, we have the following definition.

Definition 2.1. Consider a nonlinear system with input $u(t)$ and output $y(t)$ such that Assumption A1 is fulfilled. The Output Error LTI Second Order Equivalent (OE-LTI-SOE) of this system is the stable and causal LTI model $G_{0, O E}(q)$ that minimizes the mean-square error $\mathrm{E}\left((y(t)-G(q) u(t))^{2}\right)$, i.e.,

$$
G_{0, O E}(q)=\underset{G \in \mathcal{G}}{\arg \min } \mathrm{E}\left((y(t)-G(q) u(t))^{2}\right),
$$

where $\mathcal{G}$ denotes the set of all stable and causal LTI models.

The concept of LTI Second Order Equivalents has, for example, been discussed in Ljung (2001) and in Enqvist (2003). It should immediately be pointed out that the OE-LTI-SOE of a nonlinear system depends on which input signal that is used. Hence, we can only talk about the OE-LTI-SOE of a nonlinear system with respect to a particular input signal. The following theorem is a direct consequence of classic Wiener filter theory. 


\section{Theorem 2.1}

Consider a nonlinear system with input $u(t)$ and output $y(t)$ such that Assumption A1 is fulfilled. Then the Output Error LTI Second Order Equivalent (OELTI-SOE) $G_{0, O E}$ of this system is

$$
G_{0, O E}(z)=\frac{1}{r_{u} L(z)}\left[\frac{\Phi_{y u}(z)}{L\left(z^{-1}\right)}\right]_{\text {causal }},
$$

where $[. . .]_{\text {causal }}$ denotes taking the causal part and where $L(z)$ is the canonical spectral factor of $\Phi_{u}(z)$ from (1).

Proof: See, for example, Ljung (1999) or Kailath et al. (2000).

The expression (2) for the OE-LTI-SOE can be simplified in some cases. For example, the following theorem holds if the input has been generated by filtering white noise through a minimum phase filter.

\section{Theorem 2.2}

Consider a nonlinear system with input $u(t)$ and output $y(t)$ such that Assumption A1 is fulfilled. Assume that the input signal has been generated by filtering white, possibly non-Gaussian, noise $e(t)$ through a minimum phase filter $L_{m}(z)$. Assume also that any other external signals that affect the output are independent of $u$. Then the OE-LTI-SOE is

$$
G_{0, O E}(z)=\frac{\Phi_{y u}(z)}{\Phi_{u}(z)}=\frac{\Phi_{y e}(z)}{L_{m}(z) R_{e}(0)}
$$

where $R_{e}(0)=\mathrm{E}\left(e(t)^{2}\right)$.

Proof: See Enqvist (2003).

In the next section, we will use (3) to simplify the calculations of some particular OE-LTI-SOEs.

\section{$3 \quad$ Slightly Nonlinear Systems}

The use of a linear model is very natural when the true system is close to being linear. In many cases, the behavior of a slightly nonlinear system can be understood, at least intuitively, from the theory of linear systems. Hence, it is a legitimate question to ask whether this linear intuition can be extended also to OE-LTI-SOEs for slightly nonlinear systems. A slightly nonlinear system will here be defined as a system that for a certain input signal can be written

$$
y(t)=G_{l}(q) u(t)+\alpha y_{n}(t)+w(t),
$$

where the linear part, $G_{l}(q) u(t)$, of the system is much larger than the nonlinear part $\alpha y_{n}(t)$. Here, the parameter $\alpha$ defines the size of the nonlinear part of the system and $w(t)$ is a noise term.

If the nonlinear contribution to the output is small for a certain input, one might assume that the corresponding OE-LTI-SOE would be close to the linear part of the system in some sense. However, as we will see in the following example, this is not always the case. 


\section{Example 3.1}

Consider the nonlinear system

$$
\begin{aligned}
y(t) & =y_{l}(t)+\alpha y_{n}(t), \\
y_{l}(t) & =u(t), \\
y_{n}(t) & =u(t)^{3} .
\end{aligned}
$$

The output from this system consists of a linear part, $y_{l}(t)$, and a nonlinear part, $\alpha y_{n}(t)$, whose size is controlled by the parameter $\alpha$. Here, the transfer function $G_{l}(q)$ of the linear part is equal to one. For bounded input signals, small values of $\alpha$ will give a system output that is close to the output from $G_{l}$. In this sense, $\alpha$ defines how close the nonlinear system is to the linear system $G_{l}(q)$. Let the input signal be

$$
u(t)=L_{m}(q, c) e(t)
$$

where

$$
L_{m}(q, c)=\left(1-c q^{-1}\right)^{2}=1-2 c q^{-1}+c^{2} q^{-2}, \quad 0<c<1
$$

and where $e(t)$ is a white noise process with uniform distribution over the interval $[-1,1]$. For all $c$ with $0<c<1$, the input is bounded, $-4<u(t)<4$. For this input, a small value of $\alpha$ like, for example, $\alpha=0.01$ will give an output that is very similar to the output from $G_{l}$, i.e., the output when $\alpha=0$. This can be seen in Figure 2 for a particular realization of the input signal. However, the small differences between these output signals will sometimes give rise to totally different OE-LTI-SOEs.

Since the input is generated by filtering white noise through a minimum phase filter, Theorem 2.2 gives that the OE-LTI-SOE can be written

$$
G_{0, O E}(z, \alpha, c)=\frac{\Phi_{y u}(z, \alpha, c)}{\Phi_{u}(z, c)}=\underbrace{G_{l}(z)}_{=1}+\alpha \frac{\Phi_{y_{n} e}(z, c)}{L_{m}(z, c) R_{e}(0)} .
$$

If $y_{n}(t)$ is expanded we get

$$
\begin{aligned}
y_{n}(t)= & e(t)^{3}-6 c e(t)^{2} e(t-1)+3 c^{2} e(t)^{2} e(t-2)+12 c^{2} e(t) e(t-1)^{2} \\
& -12 c^{3} e(t) e(t-1) e(t-2)+3 c^{4} e(t) e(t-2)^{2}-8 c^{3} e(t-1)^{3} \\
& +12 c^{4} e(t-1)^{2} e(t-2)-6 c^{5} e(t-1) e(t-2)^{2}+c^{6} e(t-2)^{3} .
\end{aligned}
$$

Using the fact that $\mathrm{E}\left(e(t)^{2}\right)=\frac{1}{3}$ and $\mathrm{E}\left(e(t)^{4}\right)=\frac{1}{5}$ and that $e(t)$ and $e(t-k)$ are independent when $k \neq 0$, we can derive the cross-covariance function $R_{y_{n} e}(\tau, c)$

$$
\begin{aligned}
R_{y_{n} e}(0, c)= & \mathrm{E}\left(y_{n}(t) e(t)\right)=\mathrm{E}\left(e(t)^{4}\right)+12 c^{2} \mathrm{E}\left(e(t)^{2} e(t-1)^{2}\right) \\
& +3 c^{4} \mathrm{E}\left(e(t)^{2} e(t-2)^{2}\right)=\frac{1}{5}+12 c^{2} \frac{1}{9}+3 c^{4} \frac{1}{9}=\frac{1}{15}\left(3+20 c^{2}+5 c^{4}\right), \\
R_{y_{n} e}(1, c)= & \mathrm{E}\left(y_{n}(t) e(t-1)\right)=-6 c \mathrm{E}\left(e(t)^{2} e(t-1)^{2}\right)-8 c^{3} \mathrm{E}\left(e(t-1)^{4}\right) \\
& -6 c^{5} \mathrm{E}\left(e(t-1)^{2} e(t-2)^{2}\right)=-6 c \frac{1}{9}-8 c^{3} \frac{1}{5}-6 c^{5} \frac{1}{9} \\
= & -\frac{1}{15}\left(10 c+24 c^{3}+10 c^{5}\right),
\end{aligned}
$$




$$
\begin{aligned}
R_{y_{n} e}(2, c)= & \mathrm{E}\left(y_{n}(t) e(t-2)\right)=3 c^{2} \mathrm{E}\left(e(t)^{2} e(t-2)^{2}\right) \\
& +12 c^{4} \mathrm{E}\left(e(t-1)^{2} e(t-2)^{2}\right)+c^{6} \mathrm{E}\left(e(t-2)^{4}\right) \\
= & 3 c^{2} \frac{1}{9}+12 c^{4} \frac{1}{9}+c^{6} \frac{1}{5}=\frac{1}{15}\left(5 c^{2}+20 c^{4}+3 c^{6}\right) \\
R_{y_{n} e}(\tau, c)= & 0, \quad \forall \tau \in \mathbb{Z} \backslash\{0,1,2\}
\end{aligned}
$$

Inserted in (7) this gives

$$
G_{0, O E}(z, \alpha, c)=1+\alpha \frac{1}{5} \cdot \frac{b_{0}+b_{1} z^{-1}+b_{2} z^{-2}}{1-2 c z^{-1}+c^{2} z^{-2}},
$$

where

$$
\begin{aligned}
& b_{0}=3+20 c^{2}+5 c^{4}, \\
& b_{1}=-\left(10 c+24 c^{3}+10 c^{5}\right), \\
& b_{2}=5 c^{2}+20 c^{4}+3 c^{6} .
\end{aligned}
$$

Let

$$
\Delta_{G}(z, \alpha, c)=\sum_{k=0}^{\infty} \delta_{G}(k, \alpha, c) z^{-k}=G_{0, O E}(z, \alpha, c)-G_{l}(z) .
$$

Then the static gain of $\Delta_{G}$ is

$$
\Delta_{G}(1, \alpha, c)=\frac{\alpha}{5} \cdot \frac{3-10 c+25 c^{2}-24 c^{3}+25 c^{4}-10 c^{5}+3 c^{6}}{(1-c)^{2}} .
$$

From (9) we see that the numerator of $\Delta_{G}(1, \alpha, c)$ approaches $12 \alpha$ when $c \rightarrow 1$, i.e., for $c$ close to 1 we have $\Delta_{G}(1, \alpha, c) \approx \frac{12 \alpha}{5(1-c)^{2}}$. This implies that no matter how small $\alpha>0$ we select, we can always make $\Delta_{G}(1, \alpha, c)$ arbitrarily large by choosing a $c$ sufficiently close to 1 . That is, no matter how linear the system is, there is always a bounded input signal such that its OE-LTI-SOE is far from $G_{l}\left(e^{i \omega}\right)$ for $\omega=0$. The difference between $\left|G_{0, O E}\left(e^{i \omega}, 0.01,0.99\right)\right|$ and $\left|G_{l}\left(e^{i \omega}\right)\right|$ is shown in Figure 3.

Furthermore, since

$$
\left|\Delta_{G}(1, \alpha, c)\right|=\left|\sum_{k=0}^{\infty} \delta_{G}(k, \alpha, c)\right| \leq \sum_{k=0}^{\infty}\left|\delta_{G}(k, \alpha, c)\right|,
$$

it follows that for any $\alpha>0$ the $l_{1}$-norm of the impulse response of $\Delta_{G}$ can be made arbitrarily large by an appropriate choice of $c$. Furthermore, it can be shown that, for any fixed $\alpha>0$, also

$$
\sum_{k=0}^{\infty} \delta_{G}(k, \alpha, c)^{2}=\frac{1}{2 \pi} \int_{-\pi}^{\pi}\left|\Delta_{G}\left(e^{i \omega}, \alpha, c\right)\right|^{2} d \omega
$$

can be made arbitrarily large by taking a $c$ close to 1 . 


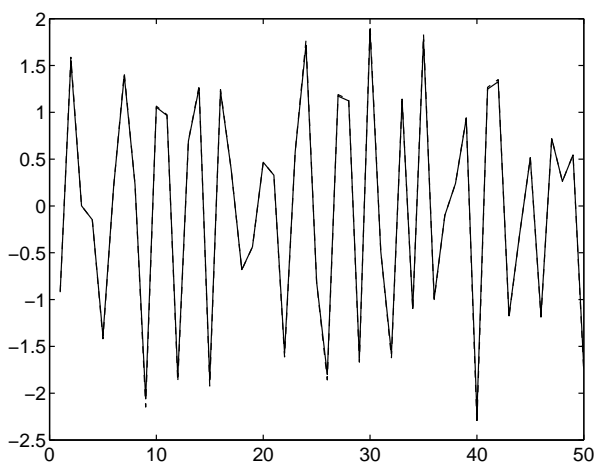

Figure 2: The output $y(t)$ (dashed) of the nonlinear system (with $\alpha=0.01$ and $c=0.99$ ) in Example 3.1 and the output $y_{l}(t)=u(t)$ (solid) of the linear part of that system cannot be distinguished for a particular realization of the input signal.

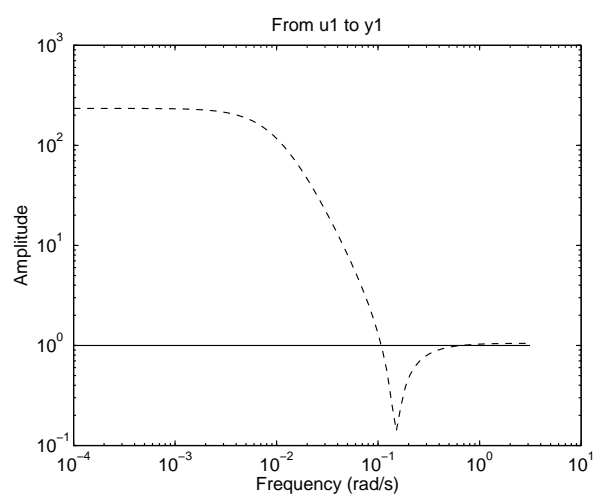

Figure 3: Bode plot showing the large difference between the OE-LTI$S O E G_{0, O E}\left(e^{i \omega}, 0.01,0.99\right)$ (dashed) and the linear part $G_{l}\left(e^{i \omega}\right)$ (solid) of the system in Example 3.1.

The previous example is a clear indication that the OE-LTI-SOE not always can be understood from linear theory. There is no corresponding behavior in the linear case, since a small linear, time-invariant deviation from $G_{l}$ in Example 3.1 only would have given rise to exactly the same small deviation in the OE-LTISOE. It should be noted that for small $\alpha$ values, $G_{l}$ is a good model of the true system and it can predict the output very well from the input signal. Despite this, the model obtained from prediction error identification will not converge to $G_{l}$ but to $G_{0, O E}$ when the number of measurements tends to infinity.

We have seen that the OE-LTI-SOE in some cases can be far from the linear part of the system. This can in some circumstances be an undesirable property, e.g. if the OE-LTI-SOE is supposed to be used as a basis for robust control design. Such a design puts restrictions on the control laws in order to guarantee the stability of the resulting true closed-loop system, despite the presence of model errors.

Assume that the true system is almost linear in the sense that it deviates 
from an LTI system $G_{l}$ by a nonlinearity with a small gain. In this case, $G_{l}$ is a very good basis for robust control design, since the small nonlinearity often only gives rise to rather mild restrictions on the controller. However, if an OELTI-SOE that is far from $G_{l}$ is used for the controller design, the restrictions on the controller might become much harder, since the gain of the model error now is large. Hence, it is interesting to investigate under what circumstances we can guarantee that the OE-LTI-SOE will be close to the linear part of the system when the nonlinearities are small.

In the case of linear undermodelling, i.e., when a linear system is approximated by a model of lower order, the optimal approximation depends only of the spectral density of the input (Ljung, 1999) and not on the distribution of the input signal components. However, when a nonlinear system is approximated with a linear model, the distribution of the input affects the result significantly. For example, in Example 3.1 the input is generated from the white uniformly distributed signal $e(t)$. If this signal is replaced with a white Gaussian signal with the same variance, the corresponding OE-LTI-SOE will be completely different. This is shown in the next example.

\section{Example 3.2}

Consider once again the nonlinear system (4) but now with an input $u(t)$ generated by filtering a white Gaussian process $e(t)$ with zero mean and variance $1 / 3$ through the filter $L_{m}(q, c)$ in (6). Since the system is static and the input is Gaussian, Theorem 1.1 gives that

$$
R_{y u}(\tau, \alpha, c)=R_{u}(\tau)+\alpha \mathrm{E}\left(3 u(t)^{2}\right) R_{u}(\tau)=\left(1+\alpha\left(1+4 c^{2}+c^{4}\right)\right) R_{u}(\tau),
$$

where we have used that $\mathrm{E}\left(u(t)^{2}\right)=\left(1+4 c^{2}+c^{4}\right) / 3$. Since (10) implies that

$$
\Phi_{y u}(z, \alpha, c)=\left(1+\alpha\left(1+4 c^{2}+c^{4}\right)\right) \Phi_{u}(z),
$$

the OE-LTI-SOE of the system is

$$
G_{0, O E}(z, \alpha, c)=\left(1+\alpha\left(1+4 c^{2}+c^{4}\right)\right)
$$

for this particular input. Hence, the fact that the input is Gaussian implies the the OE-LTI-SOE is static. Furthermore, it is obvious that the deviation of the OE-LTI-SOE from the linear part (i.e., from 1) is bounded in this case. With $\Delta_{G}(z, \alpha, c)=G_{0, O E}(z, \alpha, c)-G_{l}(z)=\alpha\left(1+4 c^{2}+c^{4}\right)$ and $0<c<1$, we have

$$
\left|\Delta_{G}(z, \alpha, c)\right|<6|\alpha| \text {. }
$$

Here, unlike in Example 3.1, we cannot make $\Delta_{G}$ large for an arbitrarily small $\alpha>0$ by selecting $c<1$ sufficiently close to 1 . For example, $\alpha=0.01$ and $c=0.99$ give

$$
G_{0, O E}(z, 0.01,0.99) \approx 1.0588
$$

and $\left|\Delta_{G}(z, \alpha, c)\right| \approx 0.0588$, which can be compared with the large $\Delta_{G}$ that is obtained when $e(t)$ is uniformly distributed (see Figure 3 ). 
From Example 3.2 we see that the fact that the input is Gaussian can, at least in some cases, prevent the OE-LTI-SOE from being far from the linear part of a slightly nonlinear system. Probably, this is often a desirable property and hence one could argue that Gaussian inputs should always be used in identification experiments where slightly nonlinear systems are approximated with LTI models. However, an input with a distribution which is similar to a Gaussian distribution might also work fine. This is illustrated in the next example.

\section{Example 3.3}

Again, consider the nonlinear system (4) with an input $u(t)$ generated by filtering a white process $e(t)$ with zero mean and variance $1 / 3$ through the filter $L_{m}(q, c)$ in (6). Let $c=0.99$ and $\alpha=0.01$. In the two previous examples, it has been shown that this choice of parameters and a uniformly distributed $e(t)$ give an OE-LTI-SOE with the frequency response shown in Figure 3 while a Gaussian $e(t)$ gives the OE-LTI-SOE in (11). Here, it will be shown that for non-Gaussian choices of $e(t)$ with distributions that are more Gaussian than the uniform distribution, the OE-LTI-SOEs will have frequency responses that are closer to the linear part of the system. Let

$$
e_{M}(t)=\frac{1}{\sqrt{M}} \sum_{k=1}^{M} \tilde{e}_{k}(t), \quad M \in \mathbb{Z}_{+},
$$

where $\tilde{e}_{k}(t)$ are independent white signals with uniform distribution over the interval $[-1,1]$ and zero mean. In this way, $\mathrm{E}\left(e_{M}(t)^{2}\right)=1 / 3$ for all $M$ and $e_{M}(t)$ will, according to the central limit theorem, become more Gaussian for larger $M$. Let

$$
u_{M}(t)=L_{m}(q, 0.99) e_{M}(t)
$$

be inputs to the nonlinear system and let the OE-LTI-SOE of the system for each of these inputs be denoted by $G_{0, O E, M}(z)$. Since all inputs have been generated in the same way as the input in Example 3.1,

$$
G_{0, O E, M}(z)=\frac{b_{0}(M)+b_{1}(M) z^{-1}+b_{2}(M) z^{-2}}{1-1.98 z^{-1}+0.9801 z^{-2}}
$$

for all $M$. Furthermore, let $u_{G}(t)$ denote the Gaussian input that is obtained when $e(t)$ is a white Gaussian input with zero mean and variance $1 / 3$ and let $G_{0, O E, G}(z)$ be the corresponding OE-LTI-SOE (i.e., the OE-LTI-SOE in (11)).

Realizations with 50000 samples of $u_{M}(t)$ for $M=1,2$ and $8, u_{G}(t)$ and of the corresponding system outputs have been generated in Matlab. Using the System Identification Toolbox, an output error model $\hat{G}_{0, O E, M}(z)$ with $n_{b}=3$, $n_{f}=2$ and $n_{k}=0$ has been estimated for each pair of input and output realizations. More specifically, the command oe (z, [3 2 0 $]$, ' lim', 0) has been used. The frequency responses of these estimated models are shown in Figure 4.

By comparing this figure with Figure 3 , it can be seen that $\hat{G}_{0, O E, 1}(z)$ indeed is close to the exact OE-LTI-SOE. Furthermore, Figure 4 shows that the OELTI-SOEs for the non-Gaussian inputs get closer to the OE-LTI-SOE for the Gaussian input if the input has a more Gaussian-like distribution. 


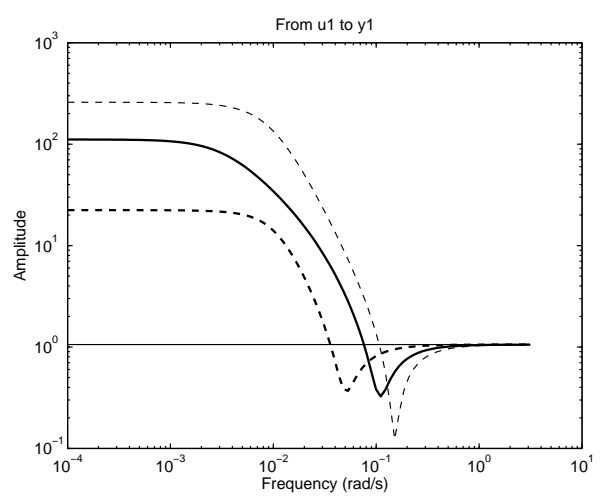

Figure 4: Bode plot showing the estimated output error models $\hat{G}_{0, O E, G}(z)$ (thin solid), $\hat{G}_{0, O E, 1}(z)$ (thin dashed), $\hat{G}_{0, O E, 2}(z)$ (thick solid) and $\hat{G}_{0, O E, 8}(z)$ (thick dashed) from Example 3.3.

The results in Example 3.3 indicate that the distance between the OE-LTISOE and the linear part of a slightly nonlinear system depends on how nonGaussian the input is. With this in mind, it seems preferable to use inputs that are as Gaussian as possible in this case.

\section{Conclusions}

In this paper, it has been shown that OE-LTI-SOEs can be very sensitive to small nonlinearities. However, from the examples presented here, it seems that this sensitivity is lower if the input is Gaussian or almost Gaussian.

\section{References}

J. S. Bendat. Nonlinear Systems Techniques and Applications. John Wiley \& Sons, New York, 1998.

S. A. Billings and S. Y. Fakhouri. Identification of systems containing linear dynamic and static nonlinear elements. Automatica, 18(1):15-26, 1982.

J. J. Bussgang. Crosscorrelation functions of amplitude-distorted Gaussian signals. Technical Report 216, MIT Research Laboratory of Electronics, Cambridge, Massachusetts, 1952.

M. Enqvist. Some results on linear models of nonlinear systems. Licentiate thesis no. 1046. Department of Electrical Engineering, Linköpings universitet, Linköping, Sweden, 2003.

I. M. Horowitz. Quantitative Feedback Design Theory. QFT Publications, Boulder, Colorado, 1993.

T. Kailath, A. H. Sayed, and B. Hassibi. Linear Estimation. Prentice Hall, Upper Saddle River, New Jersey, 2000. 
M. J. Korenberg. Identifying noisy cascades of linear and static nonlinear systems. In Proceedings of the 7th IFAC Symposium on Identification and System Parameter Estimation, pages 421-426, York, UK, 1985.

L. Ljung. Convergence analysis of parametric identification methods. IEEE Transactions on Automatic Control, 23(5):770-783, 1978.

L. Ljung. System Identification: Theory for the User. Prentice Hall, Upper Saddle River, New Jersey, second edition, 1999.

L. Ljung. Estimating linear time-invariant models of nonlinear time-varying systems. European Journal of Control, 7(2-3):203-219, 2001.

P. M. Mäkilä. Optimal approximation and model quality estimation for nonlinear systems. In Preprints of the 13th IFAC Symposium on System Identification, pages 1904-1909, Rotterdam, The Netherlands, August 2003a.

P. M. Mäkilä. Squared and absolute errors in optimal approximation of nonlinear systems. Automatica, 39(11):1865-1876, 2003b.

P. M. Mäkilä and J. R. Partington. On linear models for nonlinear systems. Automatica, 39(1):1-13, 2003.

A. Papoulis. Probability, Random Variables and Stochastic Processes. McGraw Hill, second edition, 1984.

J. R. Partington and P. M. Mäkilä. On system gains for linear and nonlinear systems. Systems \& Control Letters, 46(2):129-136, 2002.

R. Pintelon and J. Schoukens. System Identification: A Frequency Domain Approach. IEEE Press, New York, 2001.

R. Pintelon and J. Schoukens. Measurement and modelling of linear systems in the presence of nonlinear distortions. Mechanical Systems and Signal Processing, 16(5):785-801, 2002.

R. Pintelon, J. Schoukens, W. Van Moer, and Y. Rolain. Identification of linear systems in the presence of nonlinear distortions. IEEE Transactions on Instrumentation and Measurement, 50(4):855-863, 2001.

S. Sastry. Nonlinear systems - Analysis, stability and control. Springer, New York, 1999.

J. Schoukens, J. Swevers, R. Pintelon, and H. Van der Auweraer. Excitation design for FRF measurements in the presence of nonlinear distortions. In Proc. of ISMA 2002, International Conference on Noise and Vibration Engineering, pages 951-958, September 2002.

J. Schoukens, R. Pintelon, T. Dobrowiecki, and Y. Rolain. Identification of linear systems with nonlinear distortions. In Preprints of the 13th IFAC Symposium on System Identification, pages 1761-1772, Rotterdam, The Netherlands, August 2003 .

N. Wiener. Extrapolation, Interpolation and Smoothing of Stationary Time Series. Technology Press and Wiley, New York, 1949. 


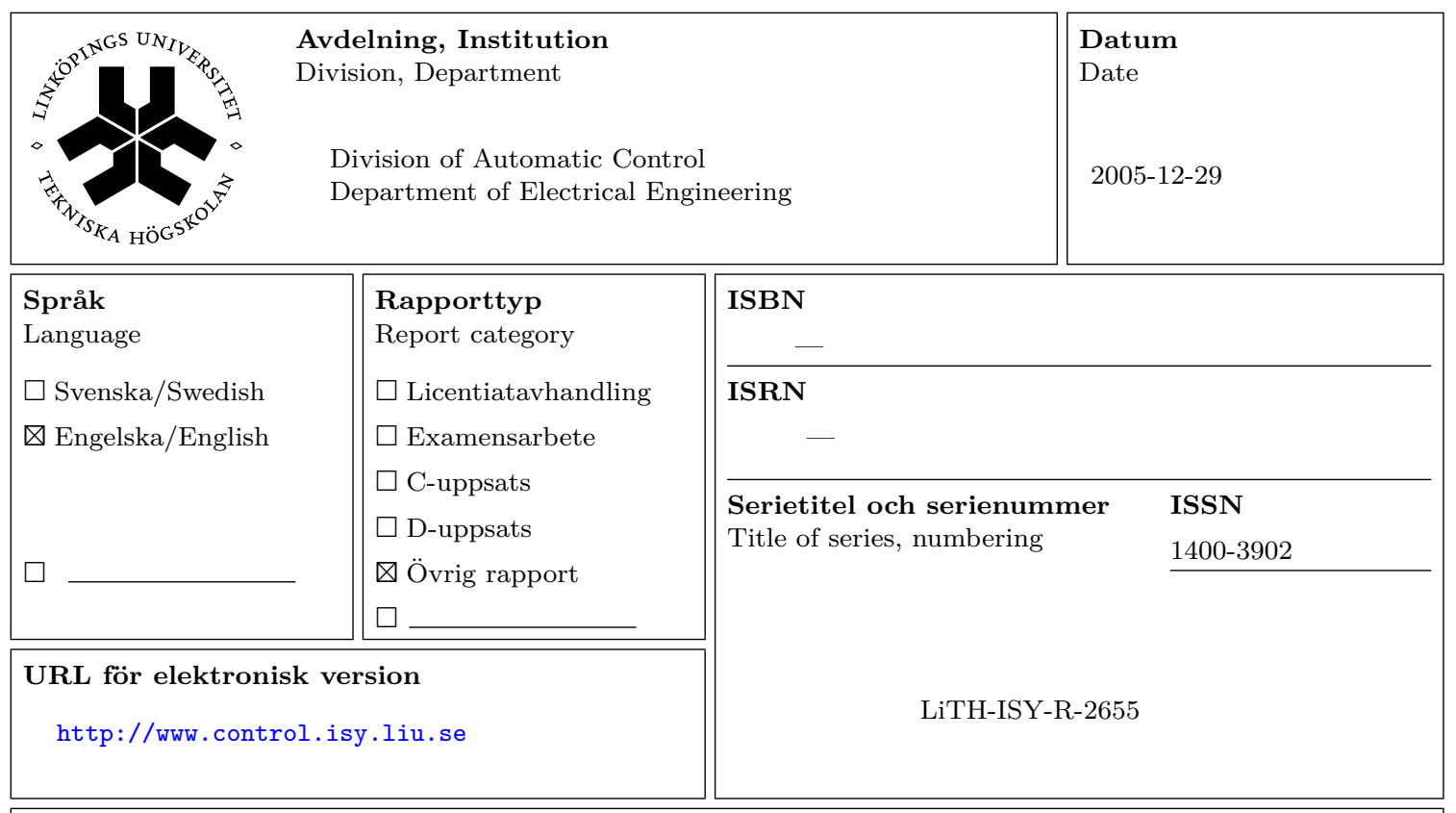

Titel LTI Approximations of Slightly Nonlinear Systems: Some Intriguing Examples

Title

Författare Martin Enqvist, Lennart Ljung

Author

\section{Sammanfattning}

Abstract

Approximations of slightly nonlinear systems with linear time-invariant (LTI) models are often used in applications. Here, LTI models that are optimal approximations in the meansquare error sense are studied. It is shown that these models can be very sensitive to small nonlinearities. Furthermore, the significance of the distribution of the input process is discussed. From the examples studied here, it seems that LTI approximations for inputs with distributions that are Gaussian or almost Gaussian are less sensitive to small nonlinearities. 